\title{
Defensive responses to threat scenarios in Brazilians reproduce the pattern of Hawaiian Americans and non-human mammals
}

\author{
R. Shuhama, C.M. Del-Ben, S.R. Loureiro and F.G. Graeff \\ Divisão de Psiquiatria, Departamento de Neurologia, Psiquiatria e Psicologia Médica, Faculdade de \\ Medicina de Ribeirão Preto, Universidade de São Paulo, Ribeirão Preto, SP, Brasil \\ Correspondence to: C.M. Del-Ben, Departamento de Neurologia, Psiquiatria e Psicologia Médica, FMRP, \\ USP, Avenida Bandeirantes, 3900, 14048-900 Ribeirão Preto, SP, Brasil \\ Fax: +55-16-3602-2544. E-mail: delben@fmrp.usp.br
}

\begin{abstract}
A former study with scenarios conducted in Hawaii has suggested that humans share with non-human mammals the same basic defensive strategies - risk assessment, freezing, defensive threat, defensive attack, and flight. The selection of the most adaptive strategy is strongly influenced by features of the threat stimulus - magnitude, escapability, distance, ambiguity, and availability of a hiding place. Aiming at verifying if these strategies would be consistent in a different culture, 12 defensive scenarios were translated into Portuguese and adapted to the Brazilian culture. The sample consisted of male and female undergraduate students divided into two groups: 76 students, who evaluated the five dimensions of each scenario and 248 medical students, who chose the most likely response for each scenario. In agreement with the findings from studies of non-human mammal species, the scenarios were able to elicit different defensive behavioral responses, depending on features of the threat. "Flight" was chosen as the most likely response in scenarios evaluated as an unambiguous and intense threat, but with an available route of escape, whereas "attack" was chosen in an unambiguous, intense and close dangerous situation without an escape route. Less urgent behaviors, such as "check out", were chosen in scenarios evaluated as less intense, more distant and more ambiguous. Moreover, the results from the Brazilian sample were similar to the results obtained in the original study with Hawaiian students. These data suggest that a basic repertoire of defensive strategies is conserved along the mammalian evolution because they share similar functional benefits in maintaining fitness.
\end{abstract}

Key words: Defensive strategies; Threat scenarios; Evolution-related responses; Lipp's Stress Symptoms Inventory for Adults

S.R. Loureiro, F.G. Graeff and R. Shuhama are recipients of fellowships from CNPq. Publication supported by FAPESP.

Received August 16, 2007. Accepted February 7, 2008

\section{Introduction}

According to the evolutionary theory of natural selection, the human species, like others, has been submitted to similar sources of natural selection that affected its evolution and shares with mammals a similar evolutionary history $(1,2)$. According to this view, basic emotions have been shaped by evolution to support decision-making processes aimed at the fulfillment of critical adaptive needs $(3,4)$. Emotional responses are mediated by unconscious information processing that allows fast reactions, highly adapted to the threatening context $(5,6)$.
The perception of threat by human beings is strongly influenced by cognitive processes, personal experience and cultural background. In spite of this, an evolutionary perspective may lead to the understanding of how modern humans cope with defense-related emotions. Firstly, a response selected to be adaptive may become faulty, as in excessive fever, which may injure tissues and provoke delirium. Secondly, there are maladaptive conditions that may be a consequence of the dissonance between behavioral tendencies inherited from our ancestors and current living conditions (7). The essentially modern human body form with elongated hind limbs dates from the Plio-Pleis- 
tocene epoch with the advent of Homo ergaster, with anatomically modern humans with modern-sized brains emerging about 200,000 years ago. Humans are likely to have retained some of the defensive and offensive adaptations of their Pleistocene ancestors, albeit, these adaptations are subject to cultural influences that mediate the context for their expression (8).

Studies of non-human species have provided substantial evidence of relatively well-defined defensive behavior patterns and of the relationship between specific defensive strategies and characteristics of the threatening circumstances to tie together the primate and rodent literature. Many non-human primates and some diurnal rodents are especially adapt at evaluating the urgency of the situation as manifested by their exhibiting immediate flight after they detect fast-moving predators or by maintaining their position on the ground to monitor slower-moving ones (cf. Refs. 5,9). Other similarities are apparent between the arboreal refugeseeking behavior of macaques, baboons and humans when they evade sprinting predators, such as lions, tigers, and leopards. Such behavior appears early in development because preschool children already exhibit precocious knowledge of how to use the environment, especially trees, to escape from a lion in experimental simulations (10).

At least five defensive strategies have been described in rodents: risk assessment, tense immobility (freezing), defensive threat, defensive attack, and flight. The selection of the most adaptive strategy depends on the dimensions of the threatening situation itself, such as the distance between the animal and the threatening stimulus, the magnitude of the threat, the certainty/uncertainty of the danger, the availability of a route of escape and/or of a hiding place (for review, see Ref. 11).

More recently, several efforts have been undertaken aiming at correlating animal defense with normal and pathological human emotions $(12,13)$. In regard to defensive behaviors, Blanchard et al. (12) have used imagined scenarios to verify if the same pattern of defensive strategies detected in non-human animal studies would be observed in human beings. Twelve scenarios, designed to vary along the five dimensions described above and known to influence animal defensive behavior, were evaluated by graduate students in Hawaii. Most of the predictions derived from the animal literature have been confirmed in this study, suggesting congruence between human and nonhuman defensive systems.

The use of scenarios allows the study of threatening situations that are difficult or unethical to experimentally reproduce in human beings, such as the investigation of behavior and physiological reactions to potential aggression (14), or sexual violence $(15,16)$. On the other hand, the scenario approach does not measure the person's actual reaction to a real situation, allowing only an inference about what the individual believes he/she might do in that situation. This can be strongly influenced by the individual's cultural background.

Considering that culturally acquired attitudes influence emotional experience $(17,18)$, the present study was aimed at verifying the pattern of behavioral responses to the defensive scenarios of a Brazilian urban university sample. The study with a population from a developing country also allows the comparison between societies with different levels of crime incidence and socioeconomic profiles.

\section{Material and Methods}

\section{Subjects}

A total of 324 students of medicine and psychology of both sexes were recruited from the Ribeirão Preto Campus of the University of São Paulo, Brazil. Exclusion criteria included history of psychiatric treatment and use of psychoactive substances. We also excluded participants who did not completely fill the research protocol. From the total sample, 76 participants (36 males, students in the fourth and fifth years of psychology or medicine; age 20 to 25 years) evaluated the dimensions of the scenarios, whereas 248 medical students (154 males, age 17 to 29 years, mean $\pm S D=21 \pm 1.98$, first to fourth year) responded to scenarios translated into Portuguese, as described below.

The study was approved by the local Research Ethics Committee and written informed consent was obtained from each participant.

\section{Assessment}

The original 12 scenarios for assessment of defensive behavior in humans proposed by Blanchard et al. (12) were adapted and translated into Portuguese by the researchers involved in this study. Based on the evaluation of the first translation of the scenarios by three independent experts, a consensus version was elaborated. This version was submitted to back-translation into the former language (English) in order to verify if the original meanings of the scenarios had been maintained. One original scenario (Coming home one day, you find an unexpected shoebox-sized package waiting for you by the mailbox. As you sit down to open it, you notice a faint ticking sound that appears to come from inside the package) that describes a very unlikely situation in Brazilian society was replaced by a situation considered as more familiar to our environment (You are alone, walking in an empty and dark place, when you hear steps just behind you). The back-translation of the final Brazilian version of the scenarios is presented below: 
Bush: You are walking alone in a familiar but isolated place when a rough looking stranger comes out from behind a tree to attack you.

Elevator: Late at night you are alone in an elevator. When it stops and the doors open, a rough looking stranger gets in fast to attack you, blocking your exit.

Stoplight: You are driving home alone in your car. While you stop at a light an irritated stranger starts to beat on the window of your car, insulting and threatening you.

Tailgating: Driving along a two-way street, you see in your rear view mirror that a car is dangerously close to yours. The driver cannot overtake you and starts to hit the horn, coming dangerously close to the back of your car.

Corner: Late at night you are walking in an unknown place. When turning a corner you accidentally bump into a man. He becomes furious and pushes you.

Acquaintance: You are in an empty place talking to somebody you do not know very well. Without an apparent motive, he/she starts to elbow and push you. You are not sure if he/she (same sex as you) is doing this seriously or is just playing with you.

Park: Late at night you are in a square when you see a rough looking stranger holding a knife, at about ten meters from you. It is obvious that he plans to attack you.

Grab: Late at night you are leaving an empty building by yourself, far from other constructions. As soon as you are outside, you feel a hand grasping your arm.

Noise: Late at night it is dark and you are sleeping alone in your bed. You suddenly wake up feeling that you heard a suspicious noise.

Phone: Late at night you are home alone preparing to go to bed when the telephone rings. You pick up, do not recognize the voice of the person, who does not identify himself and who tells you that he is in front of your house, and then hangs up.

Steps: You are walking alone in a dark and empty place when you hear steps just behind you.

Whisper: You are alone, reading a book, when you hear noises in front of your house. You cannot distinguish them well, but when you listen with more attention, it seems to be the sound of people whispering.

The attitudinal responses to these original scenarios, thought to represent highly conserved strategies of defense common to non-human mammalian species (12), were also adapted and translated into Portuguese and submitted to the same translation process described above. The possible response choices were: hide (protect him/ herself behind something, lock the doors); stand still (paralyzed with fear); flee, try to escape (run, drive fast, climb a tree); threaten to scream or call for help; yell, scream or ask for help (calling the police or somebody); threaten to at- tack; attack or fight (punch, kick, shove); investigate to see if the danger is real (observe carefully, get closer, check out); look for something to use as a weapon (a stick, a knife, a gun); apologize, negotiate or beg for mercy.

The participant had only one choice of response. When he or she considered that none of the proposed responses fulfilled the best choice criterion, he/she was asked to write down a brief report of the most likely response to the situation described in the scenario.

The five dimensions that are expected to influence the defensive behavioral strategy choice (magnitude of threat, escapability from the threat, distance between threatening stimulus and subject, ambiguity of threat source, and presence of a place of concealment or protection) were measured for each of the 12 scenarios through an interval scale varying from 1 (lowest) to 5 (highest).

With the aiming of verifying the possible interference of the participant's current emotional state in the assessment of the threatening situations depicted in the scenarios, we also applied the Lipp's Stress Symptoms Inventory for Adults (LSSI) adapted to the Brazilian population (19). This self-reported inventory evaluates stress symptoms, the type of symptom (physical and psychological), and the phase in which it is found based on criteria originated by Hans Selye: alarm, resistance, near failure, and failure. We also included questions about previous experience with situations described in the scenarios and experience with physical struggle during adult life.

\section{Procedures}

As described previously, the evaluation of the dimensions in all the scenarios was performed by a sample of 76 medical and psychological students (47.4\% males). The evaluators were asked to choose the value in a five-point scale for each of the five dimensions studied in all 12 scenarios of the questionnaire that better represents the dimension.

The possible responses to the scenarios were given by a second sample of 248 medical students (62.1\% males) who had not taken part in the assessment of the dimensions of the scenarios. They also answered the LSSI and the questions about previous experience with threatening situations and with physical struggle. The data were collected in classrooms, just after the beginning of the regular school activities.

\section{Statistical analyses}

Data were analyzed using the Statistical Package for the Social Sciences, 10th version.

The rating of the five dimensions among the 12 scenarios was analyzed by ANOVA with repeated measures, 
with one factor between subjects (sex) and two factors within subjects (12 scenarios, 5 dimensions) followed by the post-hoc Student-Newman-Keuls' test for multiple comparisons and tests of simple effects using the Student $t$-test to compare sex for each scenario. The non-parametric chisquare test was applied to analyze qualitative data as the frequency of the first-choice responses to the scenarios and to the LSSI. The Pearson product-moment correlation test was applied to investigate associations between mean values of the defensive dimensions and responses. Values of $P<0.05$ were considered statistically significant.

\section{Results}

Defensive dimensions of the scenarios

The differences among the mean ratings for each scenario were statistically significant (ANOVA with repeated measures, factor scenario $F(8.51 ; 629.54)=40.908$, $P<0.001$, interaction scenarios $x$ dimension $F(31.89$; $2360.05)=45.925, \mathrm{P}<0.001)$ ) in all five dimensions. Post hoc analyses showed that the scenarios grouped in different clusters were placed along the continuum of each dimension. Based on the Student Newman-Keuls' test, scenarios placed in the same cluster for each dimension showed mean scores without significant differences among them. The scenarios placed at the extremes of each dimension are shown in Table 1.

In the dimension magnitude of threat, the scenarios were arranged in eight clusters (Student-Newman-Keuls' test, $\mathrm{P}<0.05)$. The scenario whisper (mean $=2.10$ ) was evaluated as the least dangerous, with a significant difference from all the other scenarios, except the scenario noise $($ mean $=2.37)$. Elevator $($ mean $=4.72)$ and park (mean $=4.67$ ) were considered as the two most dangerous scenarios, and differed statistically from the remaining scenarios, but not between themselves.

In the dimension distance between threat stimulus and subject, the scenarios were clustered in four groups (Student-Newman-Keuls' test, $\mathrm{P}<0.05$ ). The scenario grab (mean $=1.49$ ) was the situation where the threat stimulus was evaluated by the participants as the closest one, and was statistically different from the remaining scenarios, except for corner (mean $=1.82$ ) and acquaintance (mean $=1.87$ ). The situation considered as most distant threat was whisper $($ mean $=3.59)$, clustered with noise $($ mean $=$ 3.57) and phone (mean $=3.34$ ).

Six clusters have been found in the dimension escapability from the threat (Student-Newman-Keuls' test, $\mathrm{P}<$ $0.05)$. Elevator (mean $=1.45$ ) was the scenario rated as the least escapable, followed by grab (mean $=2.22$ ). There are significant differences among the mean ratings of these two scenarios compared to the others. The scenario selected for the greatest escapability from the threat was stoplight (mean $=4.14)$. Post-hoc analyses have shown that the rating of this scenario was statistically higher than all other scenarios, except for whisper (mean $=3.79$ ) and tailgating (mean $=3.85)$.

In the dimension ambiguity of threat source, seven scenario clusters were found (Student-Newman-Keuls' test, $\mathrm{P}<$ 0.05 ). The scenario park (mean $=1.85$ ) was considered as the least ambiguous, whereas the scenario noise (mean = 4.03) was evaluated as the one with the most ambiguous threat. The mean rating of the scenario noise was statistically different from all other scenarios, except for whisper (mean $=3.64$ ) and acquaintance (mean $=3.59$ ).

The ratings of the scenarios in the dimension presence of a place of concealment or protection grouped in six clusters (Student-Newman-Keuls' test, $P<0.05)$. The scenarios elevator $($ mean $=1.20)$ and $g r a b($ mean $=1.99)$ were rated, respectively, as the first and second situations with the poorest chances of finding a hiding place. Both scenarios differed statistically between them and from all other scenarios. Whisper $($ mean $=4.07$ ) was evaluated as the threatening situation that offered the greatest availability of a hiding place, differing significantly from other scenarios, except for noise $($ mean $=3.75)$, stoplight $($ mean $=3.80$ ) and phone $($ mean $=3.84)$.

Sex differences in the evaluation of the scenario dimensions

Sex differences in the evaluation of the dimensions were found in several scenarios. Female volunteers considered the threats present in the scenarios bush $(\mathrm{t}(67.54)$ $=2.31, \mathrm{P}=0.02)$, corner $(\mathrm{t}(64.83)=3.15, \mathrm{P}<0.001)$, grab $(\mathrm{t}(68.75)=3.47, \mathrm{P}<0.001)$, and step $(\mathrm{t}(73.64)=2.26, \mathrm{P}=$

Table 1. Rating in a five-point scale of five dimensions for the threat scenarios.

\begin{tabular}{|c|c|c|c|c|}
\hline \multirow{2}{*}{$\begin{array}{l}\text { Dimension } \\
\text { Magnitude of threat }\end{array}$} & \multicolumn{2}{|c|}{ Scenario with the highest mean } & \multicolumn{2}{|c|}{ Scenario with the lowest mean } \\
\hline & Elevator & 4.72 & Whisper & 2.10 \\
\hline Distance between threat stimulus and subject & Whisper & 3.59 & Grab & 1.49 \\
\hline Escapability from the threat & Stoplight & 4.14 & Elevator & 1.45 \\
\hline Ambiguity of the threat & Noise & 4.03 & Park & 1.85 \\
\hline Presence of a hiding place & Whisper & 4.07 & Elevator & 1.20 \\
\hline
\end{tabular}


0.03) stronger than male volunteers. The possibility of flight in the corner scenario was considered higher by males $(\mathrm{t}(73.52)=2.63, \mathrm{P}=0.01)$, while it was rated higher by females in the park scenario $(t(73.64)=2.51, P=0.01)$. The availability of a hiding place in the acquaintance scenario was rated higher by female than male subjects $(t(72.05)=2.53, P=0.01)$. Finally, females considered the scenarios elevator $(\mathrm{t}(71.91)=2.49, \mathrm{P}=0.01)$, and grab $(\mathrm{t}(70.73)=2.56, \mathrm{P}=0.01)$ more ambiguous than males.

\section{First choice responses to the scenarios}

As shown in Table 2, the 12 scenarios were able to elicit different first-choice responses. Considering the total sample, the response fight was the most frequent in the scenarios bush, stoplight and park, and the response attack was the most frequent in the scenario elevator. In 6 scenarios (acquaintance, grab, noise, phone, whisper, and steps), the response investigate was the most frequent. The response apologize was chosen as the first choice in the scenario corner. In the scenario tailgating, the most frequent response was others.

A significant sex difference in the first choice response was found in 5 of 12 scenarios (Table 2). In the scenarios stoplight and phone, the first option was the same in both sexes, but with a statistical difference in the response rate, whereas in the scenarios elevator, grab and step, there were differences between males and females in the behavior chosen as first option.

\section{Correlation between dimensions and scenarios}

Table 3 shows significant associations $(P<0.05)$ between the response and the mean score of the five defen-

Table 2. First choice response rate to each scenario made by the total sample and by each sex of the participants.

\begin{tabular}{|c|c|c|c|c|c|c|c|c|c|}
\hline \multirow[t]{2}{*}{ Scenario } & \multicolumn{2}{|c|}{ Total sample } & \multicolumn{2}{|c|}{ Female } & \multicolumn{2}{|c|}{ Male } & \multirow[t]{2}{*}{$\chi^{2}$} & \multirow[t]{2}{*}{ d.f. } & \multirow[t]{2}{*}{$P$} \\
\hline & Behavior & $\%$ & Behavior & $\%$ & Behavior & $\%$ & & & \\
\hline Bush & Run away & $51.2 \%$ & Run away & $53.8 \%$ & Run away & $49.7 \%$ & 0.39 & 1 & 0.53 \\
\hline Elevador* & Attack & $38.3 \%$ & Yell & $30.1 \%$ & Attack & $52.9 \%$ & 55.36 & 2 & $<0.001$ \\
\hline Stoplight* & Run away & $72.2 \%$ & Run away & $80.6 \%$ & Run away & $67.1 \%$ & 5.78 & 1 & 0.02 \\
\hline Tailgating & Others & $48.0 \%$ & Others & $45.2 \%$ & Others & $49.7 \%$ & 0.47 & 1 & 0.49 \\
\hline Corner & Apologize & $63.7 \%$ & Apologize & $59.1 \%$ & Apologize & $66.5 \%$ & 1.34 & 1 & 0.25 \\
\hline Acquaintance & Check out & $65.7 \%$ & Check out & $65.6 \%$ & Check out & $65.8 \%$ & 0.00 & 1 & 0.97 \\
\hline Park & Run away & $79.0 \%$ & Run away & $75.3 \%$ & Run away & $81.3 \%$ & 1.27 & 1 & 0.26 \\
\hline Grab* & Check out & $40.7 \%$ & Freeze & $23.7 \%$ & Check out & $52.3 \%$ & 24.80 & 1 & $<0.001$ \\
\hline Noise & Check out & $73.0 \%$ & Check out & $75.3 \%$ & Check out & $71.0 \%$ & 0.54 & 1 & 0.46 \\
\hline Phone* & Check out & $51.0 \%$ & Check out & $35.5 \%$ & Check out & $60.6 \%$ & 14.73 & 1 & $<0.001$ \\
\hline Whisper & Check out & $78.0 \%$ & Check out & $77.4 \%$ & Check out & $78.7 \%$ & 0.06 & 1 & 0.81 \\
\hline Step* & Check out & $54.0 \%$ & Run away & $49.5 \%$ & Check out & $58.1 \%$ & 13.73 & 2 & $<0.001$ \\
\hline
\end{tabular}

d.f. $=$ degrees of freedom. * Statically significant sex difference $(P<0.05)$.

Table 3. Statistically significant Pearson's product moment correlations between the mean threat dimension of the scenarios and the participant's response.

\begin{tabular}{|c|c|c|c|c|c|c|c|c|}
\hline & Hide & Freeze & Run away & Threaten to scream & Yell & Attack & Check out & Look for a weapon \\
\hline \multicolumn{9}{|l|}{ Magnitude } \\
\hline Female & - & - & - & - & - & - & - & - \\
\hline Male & - & - & - & 0.58 & - & - & - & - \\
\hline \multicolumn{9}{|l|}{ Distance } \\
\hline Female & 0.66 & - & - & - & - & -0.69 & - & - \\
\hline Male & 0.79 & - & - & - & - & - & - & 0.59 \\
\hline \multicolumn{9}{|c|}{ Escapability } \\
\hline Female & - & -0.71 & - & -0.85 & -0.68 & - & - & - \\
\hline Male & - & - & - & -0.84 & - & -0.76 & - & - \\
\hline \multicolumn{9}{|l|}{ Ambiguity } \\
\hline Female & - & - & -0.61 & - & - & - & 0.88 & - \\
\hline Male & - & - & -0.69 & - & - & - & 0.91 & - \\
\hline \multicolumn{9}{|c|}{ Hiding place } \\
\hline Female & 0.59 & - & - & -0.64 & - & - & - & - \\
\hline Male & 0.61 & - & - & -0.7 & - & -0.68 & - & - \\
\hline
\end{tabular}


sive dimensions for each scenario. The response hide was positively correlated reliably with distance from the threat and the availability of a place of concealment or protection in both sexes. Among females, a low possibility of evading from the threat correlated with the behavior freeze. The certainty of the threat was associated to the option of run away in both sexes. Threaten to scream showed a relationship with low possibility of flight and the lack of a place of protection in both sexes. This behavior was also linked to the intensity of the threat, but just in male participants. Yell for help was negatively correlated to the opportunity of escaping from the threat in women. Low chance of escape and the absence of a place of protection were connected to a higher probability of attack, in male participants, while, among women, this behavioral choice was associated to the closeness of the threatening stimuli. Check out correlated with more ambiguous threats in both sexes; and look for a weapon correlated with a lower distance from the threat, but only in males.

\section{Stress symptoms}

Stress symptoms were found in 116 of 248 students (46.8\%), with 100 participants (40.2\%) fulfilling the criteria to the resistance phase of Selye's stress model. There was a statistically significant difference between the stressed and non-stressed groups in the frequencies of the first choice response only in the scenario grab $\left(\chi^{2}=12.155\right.$, d.f. $=2, P=0.002)$ and in the scenario phone $\left(\chi^{2}=5.732\right.$, d.f. $=1, P=0.017)$. Non-stressed participants tended to choose check out if the threat was real more often than stressed participants. The stressed participants distributed themselves in options associated with more urgent behaviors, such as attack and yell for help.

\section{Previous experience}

The situations described in the scenarios noise, steps and tailgating were the three most frequent threats already experienced by the participants $(68.5,50.0$ and $33.5 \%$, respectively). Participants who had had previous experiences similar to those described in the scenarios presented a significant difference in the frequency of their first-choice response in two scenarios when compared with subjects without similar experiences. Previous experience was associated to a higher proportion of run away in the scenarios stoplight ( $\chi^{2}=7.634$, d.f. $\left.=1, P=0.006\right)$ and tailgating $\left(\chi^{2}=14.170\right.$, d.f. $\left.=1, P<0.001\right)$.

There is also a statistically significant difference in the frequencies of first-choice response in two scenarios depending on a previous experience of physical struggle in adulthood (most of them, males). The history of physical combat was associated with a reliably larger proportion of participants who had chosen attack as the response to the scenario elevator $\left(\chi^{2}=12.780\right.$, d.f. $\left.=2, \mathrm{P}=0.002\right)$ and investigate to the phone scenario $\left(\chi^{2}=5.605\right.$, d.f. $=1, \mathrm{P}=$ 0.018).

\section{Discussion}

The situations presented in the 12 scenarios adapted to the Brazilian population were able to elicit different defensive responses, depending on features of the threat stimuli that are supposed to influence the choice of the defensive behavioral strategy. Moreover, the responses to the scenarios were largely in agreement with the nonhuman literature (see Refs. 9,20-23).

A comparison of our results with the Hawaiian study (12) revealed several similarities in the scenarios that occupy the extremes of each dimension evaluated. The only apparent difference found was the scenario evaluated with the lowest magnitude of threat. Nevertheless, this scenario (acquaintance) was evaluated as the least dangerous by the Hawaiian sample unlike the Brazilian sample that evaluated it as somewhat more dangerous. We have also found similarities in the most frequent first choice in each scenario. Hawaiian and Brazilian women were in agreement in 7 of 11 comparable scenarios, whereas men agreed in 8 of 11 scenarios. Cultural differences between Hawaiians and Brazilians as a function of participant sex emerged for the scenarios tailgating, corner and phone. The disagreement in the scenario grab was found only among women. Furthermore, Brazilian women showed a higher frequency of less urgent responses, such as check out, than Hawaiian women. These findings may be due to cultural influence. Since we did not investigate cultural aspects in this study, we can not specify the cultural elements that might explain these differences.

The substitution of one scenario, originally called bomb, by the scenario steps seems to be satisfactory, considering the intermediate intensity of the threat in both scenarios (Blanchard DC, personal communication). However, since the scenarios bomb and steps comprise differences in the nature of the threats, other behavioral choices might be more appropriate in the scenario steps.

The most likely response to the scenarios park, bush, and stoplight was run away, independent of the participant's sex. This urgent behavioral strategy is expected when the animal finds itself in a clear and intense threatening situation, but at a distance from the threat source and with a route of escape available $(24,25)$. The scenario park was evaluated by the participants as the second most dangerous and with the least level of ambiguity, whereas the scenario stoplight was rated as the one with largest possi- 
bility of escape.

On the other hand, defensive attack is more adapted when the situation is clearly dangerous, but there is just a short distance between the subject and the threat stimulus, and no escape route or place to hide (24). Attack was the first male choice for the scenario elevator, where the subject is trapped by the attacker. This scenario was evaluated with highest magnitude of threat, lowest possibility of escape and lowest possibility of finding a place of concealment or protection. Confirming these data, we have found that the run away response correlated with the certainty of the threat in both sexes, whereas the option among males of an attack response was associated with lower chances of escaping and with the absence of a place of protection. Interestingly, the most frequent response by females to the elevator scenario was to yell for help, which can also be considered as an effective choice, because, as social species, humans might expect help from other humans $(1,26)$.

As in reported studies with non-human species, less urgent behaviors were chosen as the preferred response to threat scenarios evaluated as less intense, more distant and more ambiguous $(21,27)$. The check out strategy, to check whether the threat is real, was the preferred response in six scenarios: whisper, noise, acquaintance, phone, steps, and grab. Sex differences have been found only in the scenarios grab and step, where the first choices of females were freeze and run away, respectively. The scenario whisperwas evaluated as the one with the largest distance from the threat, and the scenario noise as the most ambiguous. In both cases, the participants depicted themselves inside their homes, which agrees with the behaviors of both domesticated and wild rodents that run to a familiar place when threatened $(9,28)$. The ambiguity of the threat seems to be a relevant feature to elicit check out behavior. In agreement with Blanchard et al. (12), the participants' choice for this defensive response was high and positively correlated with the mean rating of the dimension ambiguity of threat source. Taking together, these data suggest a role of fundamental processes underlying responses to potential threat.

The most frequent response to the scenario cornerwas apologize, negotiate or beg for mercy. Although in this scenario the person is at a very close distance to the potential aggressor, including physical contact, it was rated with intermediate levels of threat intensity, possibility of escape, ambiguity and availability of a hiding place. The response beg for mercy may be related to the appeasement strategy that can be found in socially organized species. This strategy consists of physical postures and/or emission of vocalizations that inhibit conspecific attack (29). The appeasement/reconciliation strategy may be equivalent to behavior of non-human social species, such as the exposure to the assailant of vulnerable body parts, such as neck and belly (30), avoidance of direct eye contact $(3,10,13)$, body contact as behaviors that look like hugs, contacts mouth to mouth, grooming, and contact to genital areas (31-33). These behaviors often come together with characteristic vocalizations and facial expressions (34-36). As human beings share this defensive strategy with other mammals $(13,31)$, we may consider that human appeasement displays, such as gaze aversion, and begging for mercy, would perform the same adaptive function in the human repertoire.

Scream can also be considered as a response related to the defensive repertoire of social animals. This response does not seem to represent defensive attack, but rather the human counterpart of asking for help, because the term call for help figures in the same set of behavioral choices. A second function of screaming, analogous to the alarm calls of other primates, is pursuit deterrence in which the attacker is persuaded to give up (37).

Women tended to choose responses associated with urgent situations more often, such as yell for help, freeze, hide, and run away. These behaviors may be related to sex differences in the ability for physical struggle. The animal literature has provided evidence that, generally, males tend to fight more vigorously than females of the same species $(30,33,38)$.

The current level of stress did not seem influence the response choice, except in two scenarios (graband phone), indicating that the scenario construct is little influenced by state conditions and that the basic defensive strategies are strongly inscribed on the human defensive repertoire.

As already mentioned, an evolutionary behavioral explanation does not exclude more proximal factors, such as cultural influence and personal experience. For instance, it is not possible to disregard that sex differences reflect what is expected from women and men as socially acceptable responses, since the shared cultural values give prescriptions on what is considered desirable in each situation (39).

Hypothetical scenarios allow the study of events that are difficult to simulate or reproduce in the laboratory (38). However, the measured parameter is not the defensive behavior, per se, but what people think they would do in these situations. It is, thus, a measure of verbally revealed cognitive assessment. We cannot know for sure whether the behavioral choices have a predictive value for actual behavior. On the other hand, what people say that they would do is understood as an important part of what they will really do (40).

Despite the limitations of the present study, our data 
are in agreement with the results reported with non-human species that engage in threat appraisal, and with the working hypothesis under scrutiny. The similarities between the present study and the results of the former Hawaiian study suggest that the meaning of the original scenarios has been preserved. Finally, the results of both studies showing that the behavioral strategies chosen by humans in response to the threatening features of the scenarios were largely as predicted by the way other species deal with immediate and distant threats. Thus, it is reasonable to argue that ancestral humans were sub- jected to similar sources of natural selection for threat appraisal, a property that led to the evolution of similar defensive coping strategies

\section{Acknowledgments}

We are indebted to D. Caroline Blanchard, University of Hawaii, Honolulu, HI, USA, and to Richard G. Coss, University of California, Davis, CA, USA, for their helpful comments and suggestions.

\section{References}

1. Darwin C. The expression of emotions in man and animals. New York: Philosophical Library; 1872.

2. Dawkins R. O gene egoísta. São Paulo: Universidade de São Paulo; 1979

3. Gilbert $P$. The evolved basis and adaptive functions of cognitive distortions. Br J Med Psychol 1998; 71 (Part 4): 447 463.

4. Nesse RM. Proximate and evolutionary studies of anxiety, stress and depression: synergy at the interface. Neurosci Biobehav Rev 1999; 23: 895-903.

5. Kavaliers M, Choleris E. Antipredator responses and defensive behavior: ecological and ethological approaches for the neurosciences. Neurosci Biobehav Rev 2001; 25: 577-586.

6. Malatynska E, Knapp RJ. Dominant-submissive behavior as models of mania and depression. Neurosci Biobehav Rev 2005; 29: 715-737.

7. Williams GC. The pony fish's glow: and other clues to plan and purpose in nature. New York: Basic Books; 1998.

8. Eaton SB, Konner M, Shostak M. Stone agers in the fast lane: chronic degenerative diseases in evolutionary perspective. Am J Med 1988; 84: 739-749.

9. Hanson MT, Coss RG. Age differences in the response of California ground Squirrels (Spermophilus beecheyi) to avian and mammalian predators. J Comp Psychol 1997; 111: $174-184$

10. Coss RG, Marks S, Ramakrishnan U. Early environment shapes the development of gaze aversion by wild bonnet macaques (Macaca radiata). Primates 2002; 43: 217-222.

11. Blanchard DC, Griebel G, Blanchard RJ. Mouse defensive behaviors: pharmacological and behavioral assays for anxiety and panic. Neurosci Biobehav Rev 2001; 25: 205-218.

12. Caroline Blanchard D, Hynd AL, Minke KA, Minemoto $T$, Blanchard RJ. Human defensive behaviors to threat scenarios show parallels to fear- and anxiety-related defense patterns of non-human mammals. Neurosci Biobehav Rev 2001; 25: 761-770.

13. Misslin R. The defense system of fear: behavior and neurocircuitry. Neurophysiol Clin 2003; 33: 55-66.

14. Pietrini P, Guazzelli M, Basso G, Jaffe K, Grafman J. Neural correlates of imaginal aggressive behavior assessed by positron emission tomography in healthy subjects. Am J Psychiatry 2000; 157: 1772-1781.
15. Bernat JA, Calhoun KS, Adams HE. Sexually aggressive and nonaggressive men: sexual arousal and judgments in response to acquaintance rape and consensual analogues. J Abnorm Psychol 1999; 108: 662-673.

16. Petralia SM, Gallup Junior GG. Effects of a sexual assault scenario on handgrip strength across the menstrual cycle. Evol Hum Behav 2002; 23: 3-10.

17. Cole PM, Bruschi CJ, Tamang BL. Cultural differences in children's emotional reactions to difficult situations. Child Dev 2002; 73: 983-996.

18. Diener E, Oishi S, Lucas RE. Personality, culture, and subjective well-being: emotional and cognitive evaluations of life. Annu Rev Psychol 2003; 54: 403-425.

19. Lipp MN. Manual do Inventário de Stress para Adultos de Lipp. São Paulo: Casa do Psicólogo; 1998.

20. Wiedenmayer CP, Barr GA. Developmental changes in responsivity to threat are stimulus-specific in rats. Dev Psychobiol 2001; 39: 1-7.

21. Coss RG, Ramakrishnan U. Perceptual aspects of leopard recognition by wild bonnet macaques (Macaca radiata). Behaviour 2000; 137: 315-335.

22. Blanchard DC, Blanchard RJ. Cocaine potentiates defensive behaviors related to fear and anxiety. Neurosci Biobehav Rev 1999; 23: 981-991.

23. Ellard CG. Organization of escape movements from overhead threats in the Mongolian gerbil (Meriones unguiculatus). J Comp Psychol 1993; 107: 242-249.

24. Blanchard RJ, Flannelly KJ, Blanchard DC. Defensive behavior of laboratory and wild Rattus norvegicus. J Comp Psychol 1986; 100: 101-107.

25. Blanchard RJ, Hebert MA, Ferrari PF, Palanza P, Figueira $\mathrm{R}$, Blanchard DC, et al. Defensive behaviors in wild and laboratory (Swiss) mice: the mouse defense test battery. Physiol Behav 1998; 65: 201-209.

26. Lummaa V, Vuorisalo T, Barr RG, Lehtonen L. Why cry? Adaptive significance of intensive crying in human infants. Evol Hum Behav 1998; 19: 193-202.

27. Dielenberg RA, McGregor IS. Defensive behavior in rats towards predatory odors: a review. Neurosci Biobehav Rev 2001; 25: 597-609.

28. Ellard CG. Laboratory studies of antipredator behavior in the Mongolian gerbil (Meriones unguiculatus): factors af- 
fecting response attenuation with repeated presentations. $J$ Comp Psychol 1996; 110: 155-163.

29. Maier R. Competition, aggression, and peacemaking. In: Maier R (Editor), Comparative animal behavior: an evolutionary and ecological approach. Boston: Allyn and Bacon; 1998. p 298-319.

30. Panksepp J. Nature red in tooth and claw: the neurobiological sources of rage and anger. In: Panksepp J (Editor), Affective neuroscience: the foundations of human and animal emotions. New York: Oxford University; 1998. p 187205.

31. de Waal FB. The integration of dominance and social bonding in primates. Q Rev Biol 1986; 61: 459-479.

32. de Waal FB. Primates - a natural heritage of conflict resolution. Science 2000; 289: 586-590.

33. Wrangham R, Peterson D. Demonic males: apes and the origins of human violence. Boston: Houghton Mifflin; 1996.

34. Cheney DL, Seyfarth RM. Reconciliatory grunts by domi- nant female baboons influence victims' behaviour. Anim Behav 1997; 54: 409-418.

35. de Waal FB. Darwin's legacy and the study of primate visual communication. Ann N Y Acad Sci 2003; 1000: 7-31.

36. Goodall J. In the shadow of man. Boston: Houghton Mifflin; London: Collins; 1971.

37. Zuberbühler K, Jenny D, Bshary R. The predator deterrence function of primate alarm calls. Ethology 1999; 105: 477490.

38. Gilbert P. Evolutionary psychopathology: why isn't the mind designed better than it is? Br J Med Psychol 1998; 71 (Part 4): 353-373.

39. Rapee RM. Perceived threat and perceived control as predictors of the degree of fear in physical and social situations. J Anxiety Disord 1997; 11: 455-461.

40. Daly M, Wilson MI. Human evolutionary psychology and animal behaviour. Anim Behav 1999; 57: 509-519. 